\title{
Osteodifferentiated Mesenchymal Stem Cells from Bone Marrow and Adipose Tissue Express HLA-G and Display Immunomodulatory Properties in HLA-Mismatched Settings: Implications in Bone Repair Therapy
}

\author{
Florent Montespan, ${ }^{1,2}$ Frédéric Deschaseaux, ${ }^{3}$ Luc Sensébé, ${ }^{3}$ \\ Edgardo D. Carosella, ${ }^{1,2}$ and Nathalie Rouas-Freiss ${ }^{1,2}$ \\ ${ }^{1}$ CEA, Institut des Maladies Emergentes et des Therapies Innovantes (IMETI), Service de Recherche en Hemato-Immunologie (SRHI), \\ Hopital Saint-Louis, IUH, 1 avenue Claude Vellefaux, 75010 Paris, France \\ ${ }^{2}$ Universite Paris Diderot, Sorbonne Paris Cité, IUH, Hopital Saint-Louis, UMR_E5, IUH, 1, avenue Claude Vellefaux, \\ 75010 Paris, France \\ ${ }^{3}$ Stromalab UMR UPS/CNRS 5273, EFS-Pyrénées-Méditerranée, U1031 Inserm, 31432 Toulouse, France
}

Correspondence should be addressed to Nathalie Rouas-Freiss; nathalie.rouas-freiss@cea.fr

Received 10 January 2014; Accepted 10 March 2014; Published 30 April 2014

Academic Editor: Fabio Morandi

Copyright (C) 2014 Florent Montespan et al. This is an open access article distributed under the Creative Commons Attribution License, which permits unrestricted use, distribution, and reproduction in any medium, provided the original work is properly cited.

\begin{abstract}
Mesenchymal stem cells (MSCs) are multipotent cells that can be obtained from several sources such as bone marrow and adipose tissue. Depending on the culture conditions, they can differentiate into osteoblasts, chondroblasts, adipocytes, or neurons. In this regard, they constitute promising candidates for cell-based therapy aimed at repairing damaged tissues. In addition, MSCs display immunomodulatory properties through the expression of soluble factors including HLA-G. We here analyse both immunogenicity and immunosuppressive capacity of MSCs derived from bone marrow and adipose tissue before and after osteodifferentiation. Results show that HLA-G expression is maintained after osteodifferentiation and can be boosted in inflammatory conditions mimicked by the addition of IFN- $\gamma$ and TNF- $\alpha$. Both MSCs and osteodifferentiated MSCs are hypoimmunogenic and exert immunomodulatory properties in HLA-mismatched settings as they suppress $\mathrm{T}$ cell alloproliferation in mixed lymphocyte reactions. Finally, addition of biomaterials that stimulate bone tissue formation did not modify MSC immune properties. As MSCs combine both abilities of osteoregeneration and immunomodulation, they may be considered as allogenic sources for the treatment of bone defects.
\end{abstract}

\section{Introduction}

Bone is among the most frequently transplanted tissues with about 1 million procedures annually in Europe. Despite their considerable disadvantages, including the risk of disease transfer and immunologic rejection, limited supply of bone, costs, and complications, allografts and autografts account for more than $80 \%$ of total graft volume. Significant growth opportunities exist for synthetic bone grafts in association with mesenchymal stem cells (MSCs) from autologous or allogenic sources as alternatives to biological bone grafts in orthopaedic and maxillofacial surgery $[1,2]$. In a classical approach, bone tissue engineering consists of harvesting bone marrow from a patient, isolating MSCs by their adherence to tissue culture plastic, expanding and differentiating those cells in culture to a sufficient number, and then seeding them onto a suitable synthetic scaffold prior to implantation into the same patient [3].

MSCs can be isolated from different tissues including bone marrow (BM), adipose tissue (AT), and perinatal sources [4]. Many reports highlighted the immunomodulatory properties of MSCs relying on three 

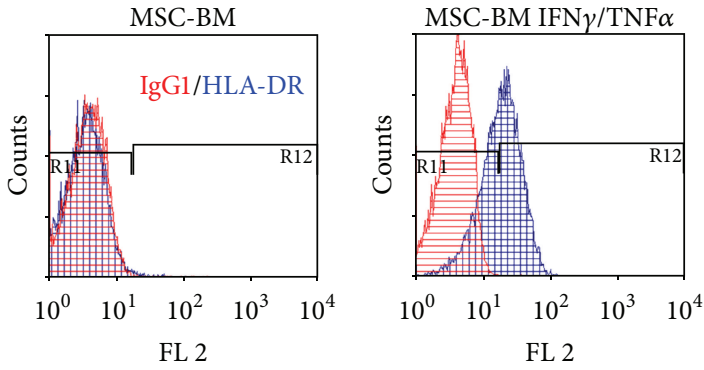

(a)

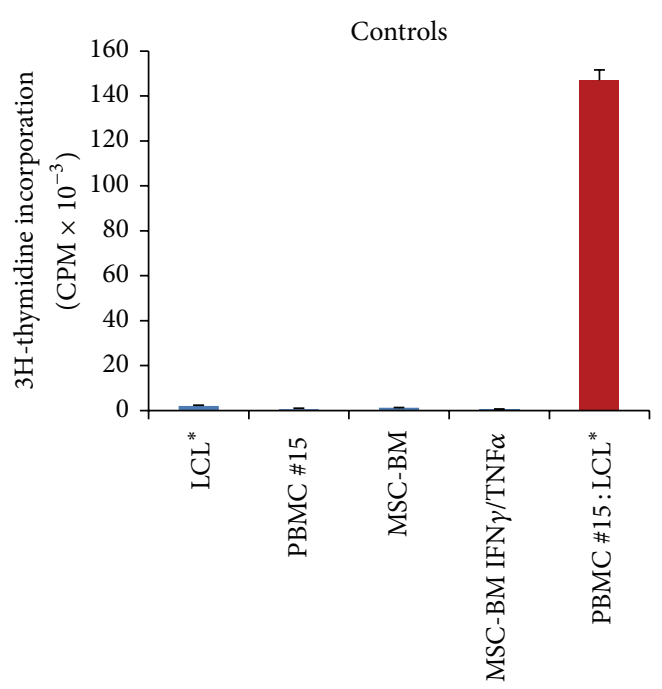

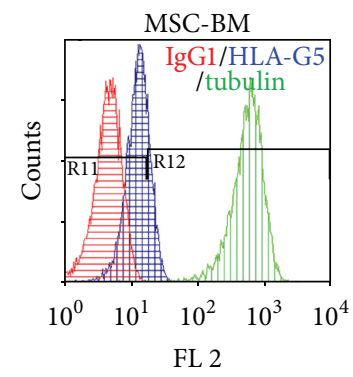

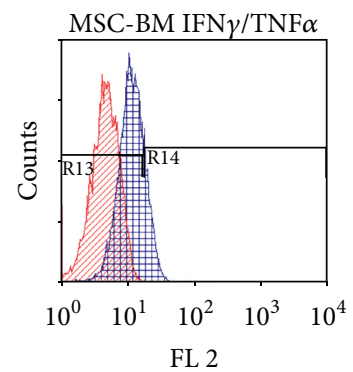

(b)



(c)

FIGURE 1: BM-derived MSCs express HLA-G and are hypoimmunogenic. (a) Expression of HLA-DR molecules was evaluated by flow cytometry analysis on BM-derived MSCs pretreated with IFN $\gamma$ and TNF $\alpha$ (MSC-BM IFN $\gamma / \mathrm{TNF} \alpha$ ) or not (MSC-BM). IgG1 was used as isotype control Ab. (b) Expression of HLA-G5 was evaluated by intracellular flow cytometry analysis on BM-derived MSCs pretreated with IFN $\gamma$ and TNF $\alpha$ (MSC-BM IFN $\gamma /$ TNF $\alpha$ ) or not (MSC-BM). Tubulin was used as positive control of cell permeabilization. (c) PBMC from healthy individual (\#15) were used as responder cells towards BM-derived MSCs pretreated with IFN $\gamma$ and TNF $\alpha$ (MSC-BM IFN $\gamma /$ TNF $\alpha$ ) or not (MSC-BM) as stimulating cells at various responder:stimulator ratios. Irradiated LCL ${ }^{*}$ were used as positive control of $\mathrm{T}$ cell alloproliferation. Results are given as mean $\mathrm{cpm} \pm$ s.e.m.; one representative experiment is shown.

main mechanisms: (1) cell cycle arrest of immune cells at the G1 phase, (2) direct interaction with immune cells, and (3) paracrine effect through secretion of various factors including HLA-G, prostaglandin E2, cytokines (TGF $\beta$, IL6, IL10, HGF, VEGF, etc.), and enzymes (indoleamine 2,3-dioxygenase and inducible nitric oxide synthase) [5-8]. Based on these tolerogenic properties, allogenic MSCs are currently tested in various clinical trials $[9,10]$.

HLA-G molecules expressed by mesenchymal stem cells fulfill an important function since blockade of HLA-G using HLA-G neutralizing antibodies could reverse MSC ability to (i) generate in vitro the expansion of $\mathrm{CD} 4^{+} \mathrm{CD} 25^{+} \mathrm{FoxP}^{+}$ regulatory $\mathrm{T}$ cells, (ii) inhibit the alloproliferative $\mathrm{T}$ cell response, and (iii) suppress the cytotoxic function of NK cells. These results show that HLA-G molecules, mainly soluble HLA-G5, actively contribute to the immunosuppressive properties exerted by MSCs [11, 12].

In clinical trials aimed at repairing bone defects, the main objective is to develop new biomaterials that simulate bone issue formation in combination with MSCs. In this context, our work entailed assessing, from an immunological perspective, whether allogenic MSCs could be used without a risk of rejection instead of autologous MSCs. The results obtained in vitro validate this hypothesis since the MSCs proved to be hypoimmunogenic and immunosuppressive in allogenic conditions. Moreover, following infusion in bone, MSCs may undergo osteodifferentiation process under the influence of in vivo osteogenic factors. We thus evaluated whether (1) allogenic MSCs committed to osteodifferentiation process can be rejected or not due to histoincompatibility and (2) combination with biomaterials modifies MSCs immune properties.

\section{Materials and Methods}

2.1. Isolation of PBMC. PBMC were isolated from blood of healthy volunteer donors (after informed consent) from the French Blood Establishment (EFS, Saint-Louis Hospital, 


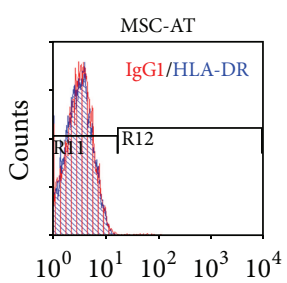

FL 2

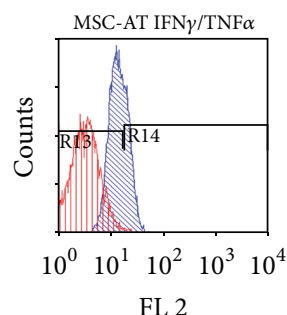

(a)

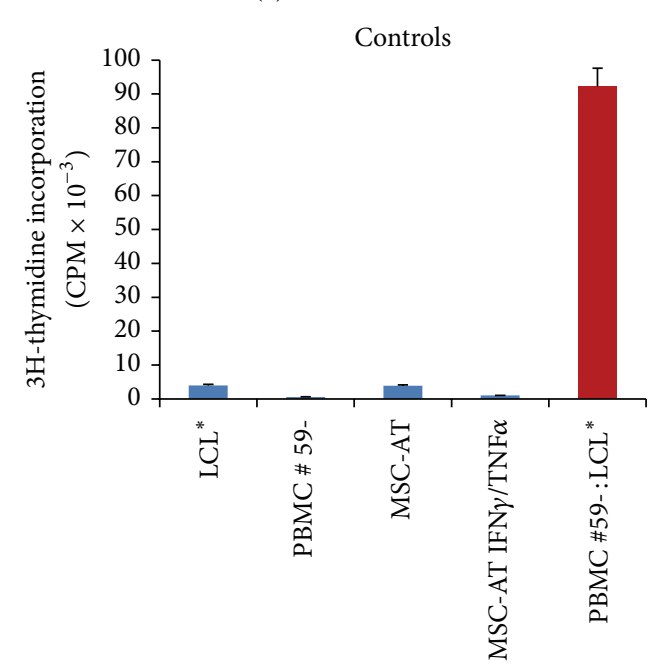

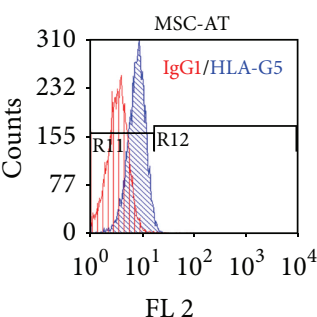

FL 2

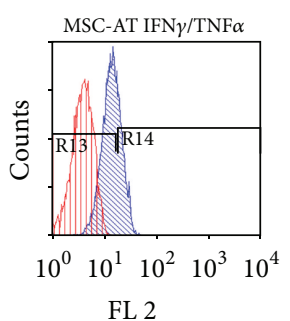

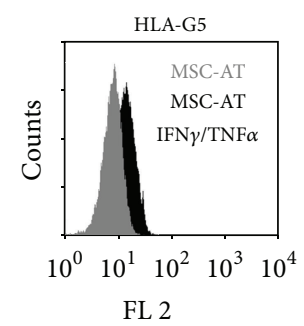

FL 2

(b)

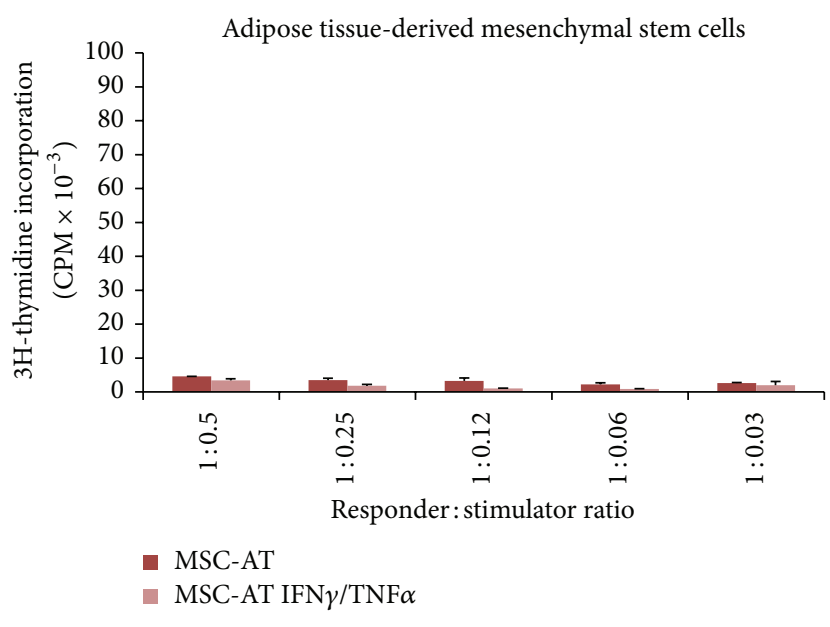

(c)

Figure 2: AT-derived MSCs express HLA-G and are hypoimmunogenic. (a) Expression of HLA-DR molecules was evaluated by flow cytometry analysis on AT-derived MSCs pretreated with IFN $\gamma$ and TNF $\alpha$ (MSC-AT IFN $\gamma /$ TNF $\alpha$ ) or not (MSC-AT). IgG1 was used as isotype control Ab. (b) Expression of HLA-G5 was evaluated by intracellular flow cytometry analysis on AT-derived MSCs pretreated with IFN $\gamma$ and TNF $\alpha$ (MSC-AT IFN $\gamma /$ TNF $\alpha$ ) or not (MSC-AT). (c) PBMC from healthy individual (\#59-) were used as responder cells towards AT-derived MSCs pretreated with IFN $\gamma$ and TNF $\alpha$ (MSC-AT IFN $\gamma / \mathrm{TNF} \alpha$ ) or not (MSC-AT) as stimulating cells at various responder : stimulator ratios. Irradiated $\mathrm{LCL}^{*}$ were used as positive control of T cell alloproliferation. Results are given as mean cpm \pm s.e.m.; one representative experiment is shown.

Paris, France) by density-gradient centrifugation over FicollPaque PLUS (GE Healthcare). These cells were used as HLAmismatched responding cells in MLR.

2.2. In Vitro Osteodifferentiation. MSCs from BM or AT were obtained from Reborne consortium center (http://www.reborne.org/). Before experiments, MSCs were thawed and expanded through seeding 1000 cells $/ \mathrm{cm}^{2}$ in T75 flasks. When cultures reach $60-70 \%$ confluence cells were harvested and seeded for immunological assays. The osteodifferentiated MSCs used as stimulating cells in MLR were obtained as previously described [13]. Briefly, MSCs were cultured in osteoblastic differentiation medium consisting of $\alpha$ MEM, FBS, ascorbic acid, $\mathrm{NaH}_{2} \mathrm{PO}_{4}$, and BMP-4 during 14 to 23 days.

2.3. Preparation of MSC-Biomaterial Complex For Immunological Tests. 24-well (ultralow attachment) plates containing disks of $\mathrm{MBCP}+$ (macroporous biphasic calcium phosphate) granules with the same diameter of each well bottom were prepared in collaboration with Biomatlante (France) to achieve a complete adherence of seeded MSCs to the biomaterial and then a complete induction of osteoblastic differentiation of MSCs. The disks of MBCP+ were washed for 48 hours before use to avoid any toxic effect on MSCs. Then MSCs were seeded on MBCP discs during 5 days in order to colonize homogenously the particles. Then, MSC-biomaterial complex was tested in mixed lymphocyte reactions.

2.4. Mixed Lymphocyte Reaction (MLR) Using MSC as Either Stimulating Cells Or Third-Party Cells Facing HLAMismatched PBMC as Responder Cells. PBMC from different donors were used as responder cells and MSCs or osteodifferentiated MSCs from BM or AT were used as either allogenic stimulating cells or third-party cells at various ratios. The HLA class II $^{+}$human B-lymphoblastoid cell line LCL 721.221 (ATCC) was used as positive control to stimulate HLAmismatched PBMC. When LCL cells were used as stimulator cells, a 75 Gy dose irradiation was given and responder: stimulator ratio was of $1: 0.5$ with a final concentration of PBMC responder cells of $10^{5}$ cells/well in a 96-well flat bottomed plate. 




FL 2

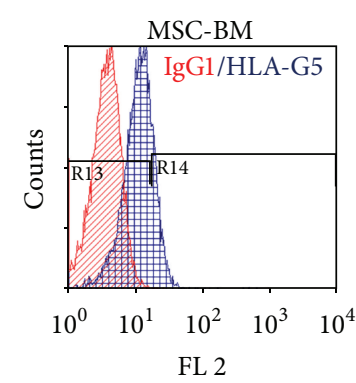

(a)

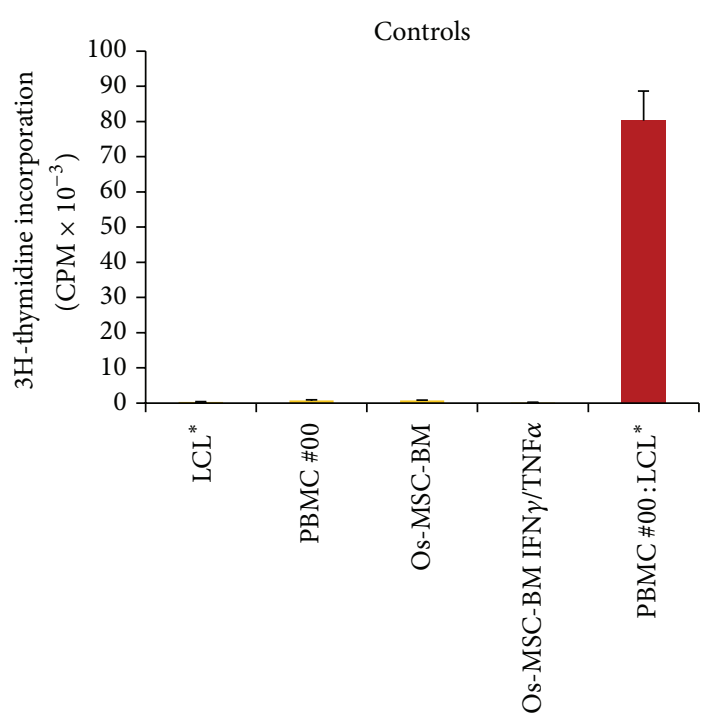

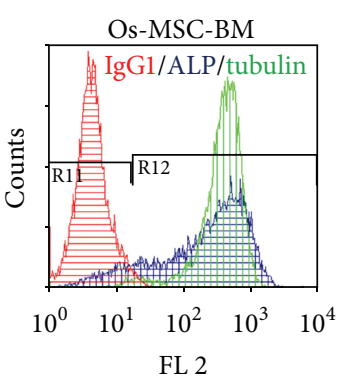

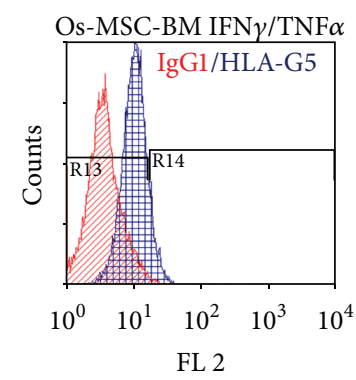

(b)

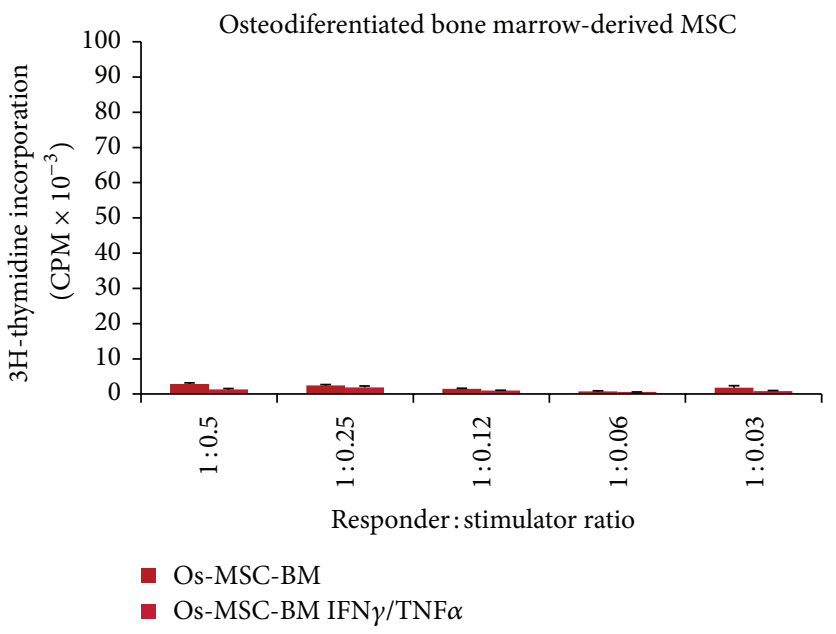

(c)

FIGURE 3: BM-derived MSCs committed to osteodifferentiation process express HLA-G and are hypoimmunogenic. (a and b) Expression of ALP and HLA-G5 was evaluated by intracellular flow cytometry analysis on BM-derived MSCs committed to 14-day osteodifferentiation process and pretreated with IFN $\gamma$ and TNF $\alpha$ (Os-MSC-BM IFN $\gamma /$ TNF $\alpha$ ) or not (Os-MSC-BM). IgG1 was used as isotype control Ab. Tubulin was used as positive control of cell permeabilization. (c) PBMC from healthy individual (\#00) were used as responder cells towards BMderived MSCs committed to osteodifferentiation process and pretreated with IFN $\gamma$ and TNF $\alpha$ (Os-MSC-BM IFN $\gamma /$ TNF $\alpha$ ) or not (Os-MSC$\mathrm{BM})$ as stimulating cells at various responder: stimulator ratios. Irradiated $\mathrm{LCL}^{*}$ were used as positive control of T cell alloproliferation. Results are given as mean cpm \pm s.e.m.; one representative experiment is shown.

Particularly, in MBCP experiments, MLR were performed in 24-well bottomed plates with a final concentration of PBMC responder cells of $10^{6}$ cells/well. Experiments comparing the effects of standard culture condition (no MBCP) and three-dimensional culture setting using MBCP discs were both performed in 24-well bottomed plates.

Cultures were incubated at $37^{\circ} \mathrm{C}$ in a humidified $5 \%$ $\mathrm{CO}_{2}$ air atmosphere. PBMC proliferation was measured at day 6 by $\left[{ }^{3} \mathrm{H}\right]$-thymidine incorporation $(1 \mu \mathrm{Ci} /$ well, Perkin Elmer) during the last 18 hours of culture. Cells were then harvested on filtermats A and thymidine incorporation into DNA was quantified, using a beta counter (Wallac 1450, Pharmacia). All samples were run in triplicate. The influence of MSC licensing with inflammatory cytokines such as IFN- $\gamma$ at $10 \mathrm{ng} / \mathrm{mL}$ (Peprotech) and plus TNF- $\alpha$ at $15 \mathrm{ng} / \mathrm{mL}$ (R\&D systems) was analyzed by adding these cytokines in cultures 48 hours before MLR [14].

2.5. Flow Cytometry. The cytokine (IFN- $\gamma$ and TNF- $\alpha$ ) treatment efficiency was checked by cytofluorometry analysis through the upregulation of HLA-DR expression on MSC. Briefly, cells were washed in PBS and stained with the anti-HLA-DR conjugated with PE (Beckman Coulter) in PBS 2\% heat-inactivated fetal calf serum for 30 minutes at $4^{\circ} \mathrm{C}$. Control aliquots were stained with an isotype-matched antibody to evaluate nonspecific binding to target cells. Fluorescence was detected by using the Epics XL4 flow cytometer (Beckman Coulter, Brea, CA, USA).

The expression of the HLA-G5 soluble isoform by MSCs was assessed by using the $2 \mathrm{~A} 12 \mathrm{mAb}$ (Exbio) after cell permeabilization. The osteodifferentiation process was verified by cytofluorometry analysis through the induction of alkaline phosphatase (ALP) expression in osteodifferentiated MSC after cell permeabilization. Briefly, cells were first permeabilized by using saponin (Sigma) and then stained with $2 \mathrm{~A} 12$ or anti-ALP (R\&D systems) for 30 minutes at $4^{\circ} \mathrm{C}$. After washing, cells were subsequently stained with an $\mathrm{F}\left(\mathrm{ab}^{\prime}\right) 2$ goat anti-mouse IgG antibody conjugated with PE (Beckman Coulter) for 30 minutes at $4^{\circ} \mathrm{C}$. Control aliquots were stained with an isotype-matched antibody to evaluate nonspecific 




(a)

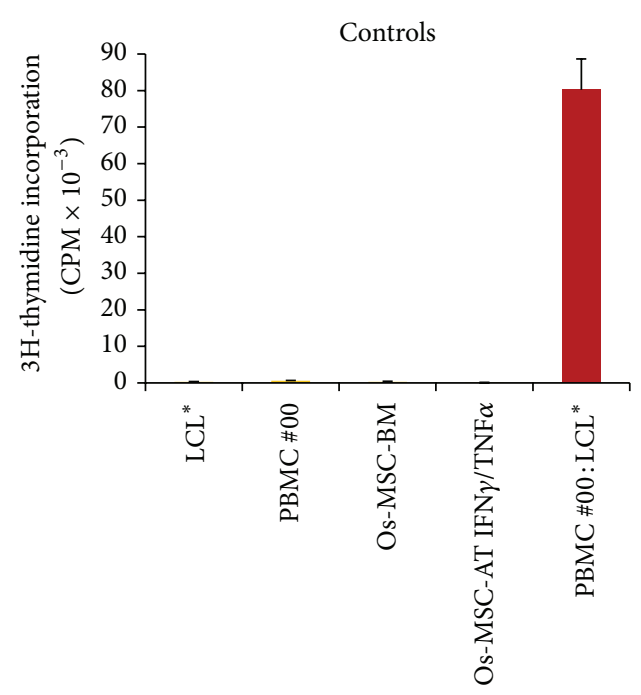



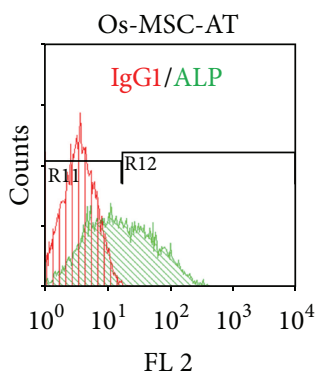

FL 2

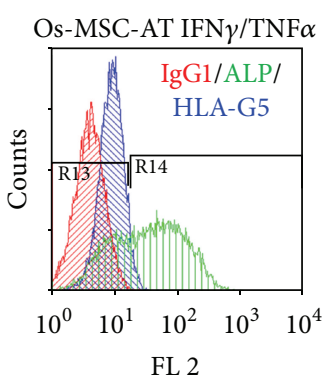

(b)

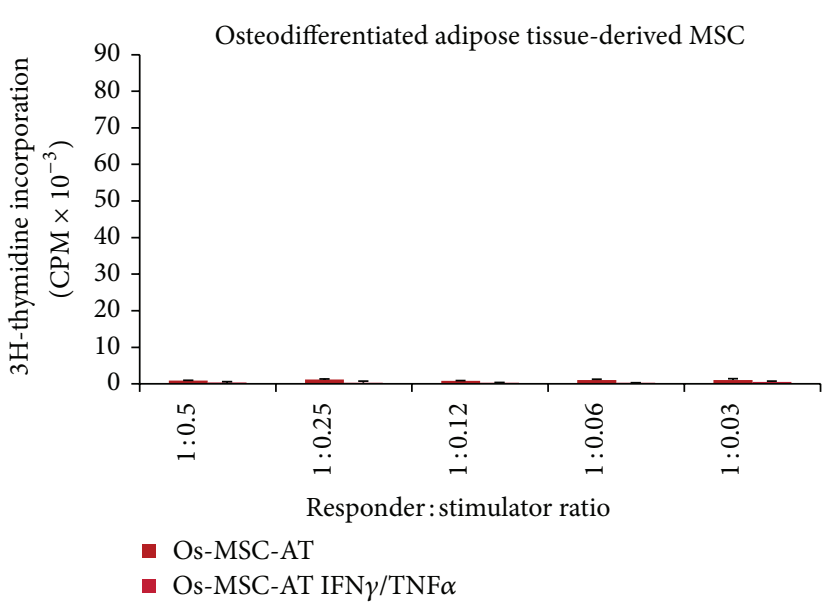

(c)

Figure 4: AT-derived MSCs committed to osteodifferentiation process express HLA-G and are hypoimmunogenic. (a) Expression of HLADR molecules was evaluated by flow cytometry analysis on AT-derived MSCs committed to 14-day osteodifferentiation process and pretreated with IFN $\gamma$ and TNF $\alpha$ (Os-MSC-AT IFN $\gamma / \mathrm{TNF} \alpha$ ) or not (Os-MSC-AT). (b) Expression of ALP and HLA-G5 was evaluated by intracellular flow cytometry analysis on AT-derived MSCs committed to 14-day osteodifferentiation process and pretreated with IFN $\gamma$ and TNF $\alpha$ (OsMSC-AT IFN $\gamma / \mathrm{TNF} \alpha$ ) or not (Os-MSC-AT). IgG1 was used as isotype control Ab. (c) PBMC from healthy individual (\#00) were used as responder cells towards AT-derived MSCs committed to osteodifferentiation process and pretreated with IFN $\gamma$ and TNF $\alpha$ (Os-MSC-AT IFN $\gamma / \mathrm{TNF} \alpha$ ) or not (Os-MSC-AT) as stimulating cells at various responder : stimulator ratios. Irradiated LCL ${ }^{*}$ were used as positive control of $\mathrm{T}$ cell alloproliferation. Results are given as mean $\mathrm{cpm} \pm$ s.e.m.; one representative experiment is shown.

binding to target cells. Fluorescence was detected by using the Epics XL4 flow cytometer.

2.6. Statistical Analysis. Significance was assessed by using a nonparametric Mann-Whitney test, assuming a $P$ value $<$ 0.05 as significant, and was marked with $*$ in the figures.

\section{Results and Discussion}

Two main questions were addressed in the present study relying on whether immune regulatory properties of MSCs are modified by (1) MSC differentiation towards the osteoblastic cell lineage or (2) the addition of synthetic biomaterial (i.e., $\mathrm{MBCP}+$ granules). In this regard, we analyzed both the immunogenic and immunosuppressive properties of MSCs from BM or AT in allogenic conditions, that is, facing HLAmismatched PBMC. To identify low immunogenic MSC types, we studied their ability to be recognized as allogenic cells by HLA-mismatched PBMC in MLR using MSCs as stimulating cells and PBMC from various healthy donors as responder cells. To examine their immunosuppressive properties, we studied their ability to modulate T cell alloproliferation as third-party cells in a classical MLR. All the functional experiments were performed by considering the differentiation status of MSC, either immature or osteodifferentiated, and seeded onto biomaterial or not.

No PBMC alloproliferation was observed in response to various doses of allogenic MSCs derived from bone marrow (Figure 1(c)) or adipose tissue (Figure 2(c)) even after licensing with IFN- $\gamma$ and TNF- $\alpha$ (Figures $1(\mathrm{c})$ and $2(\mathrm{c})$ ). Tables 1 and 2 summarize the results obtained with PBMC from distinct healthy donors. The efficiency of cytokine treatment was attested by the induction of HLA-DR expression on MSCs (Figures 1(a) and 2(a)). In order to evaluate the influence of osteodifferentiation process on the immunogenicity of MSCs, similar functional assays were performed using BMderived and AT-derived MSCs committed to preosteoblastic 

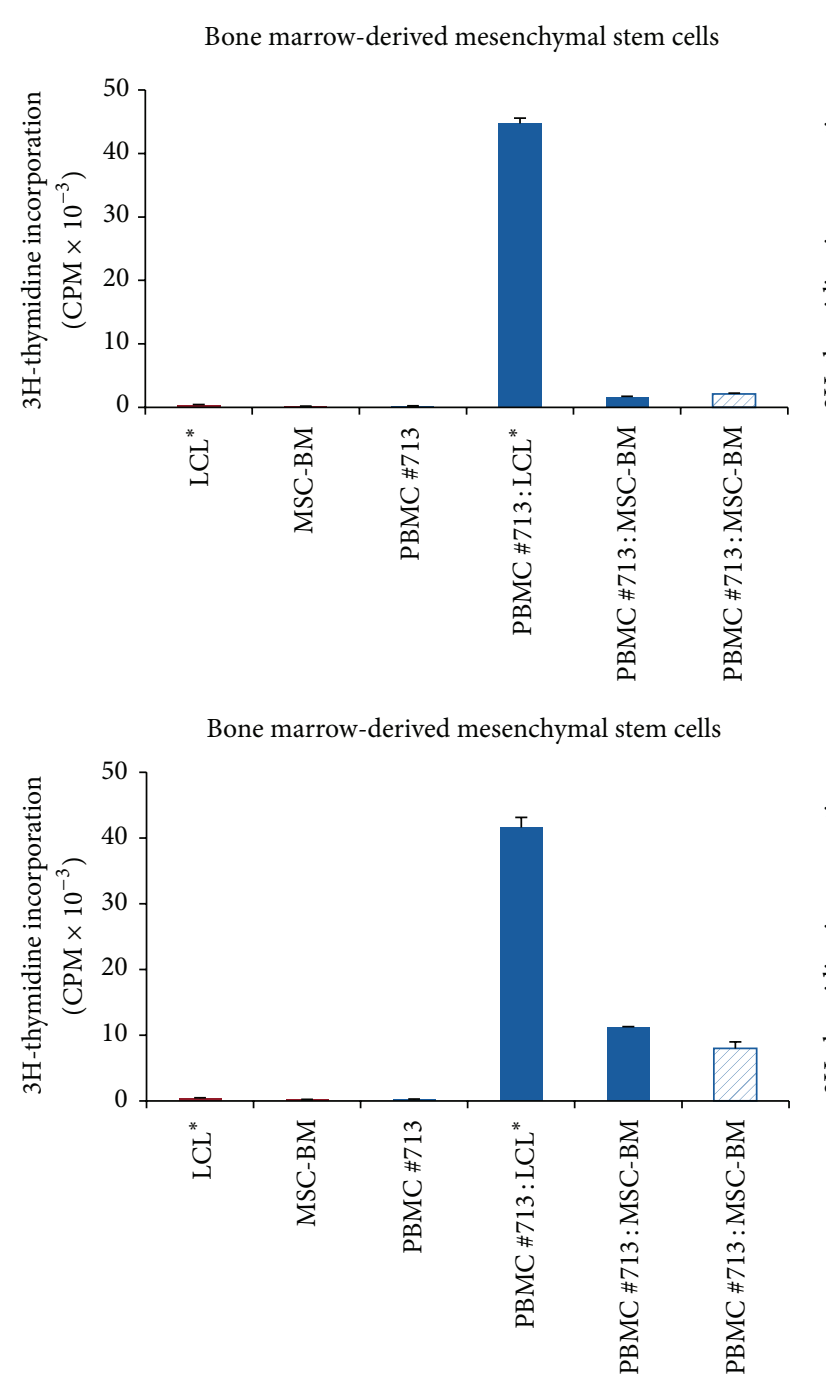

$+\mathrm{IFN} \gamma / \mathrm{TNF} \alpha$
Adipose tissue-derived mesenchymal stem cells

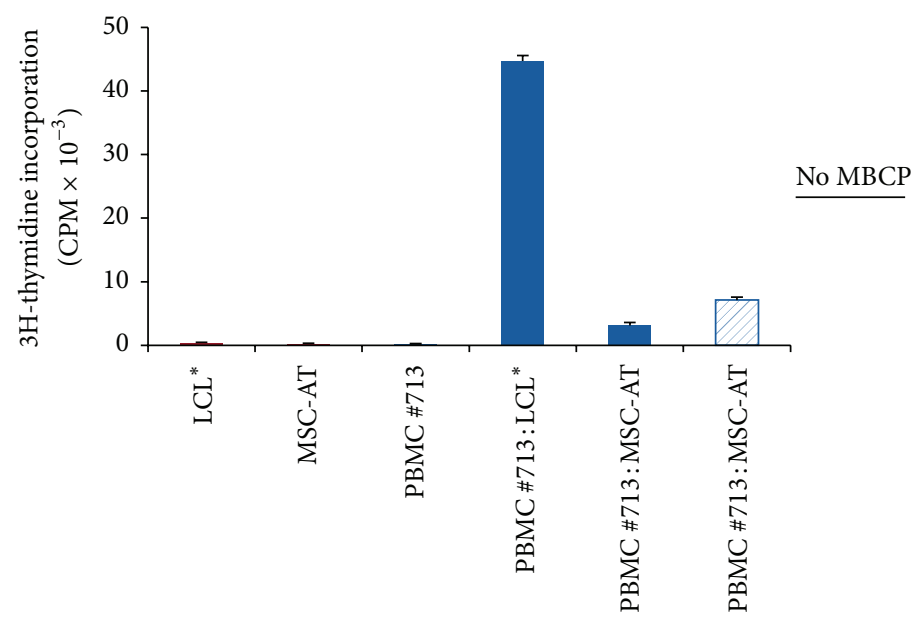

Adipose tissue-derived mesenchymal stem cells

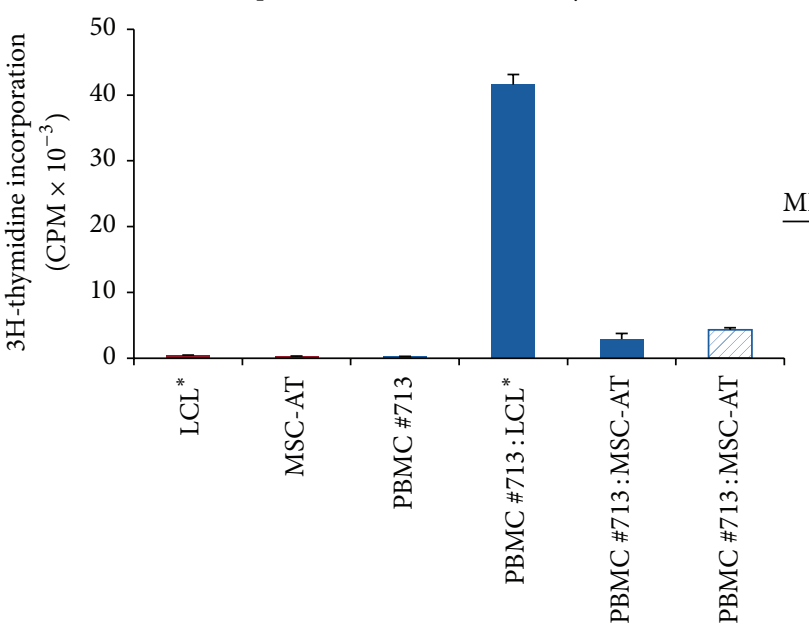

$+\mathrm{IFN} \gamma / \mathrm{TNF} \alpha$

FIgure 5: Both BM- andAT-derived MSCs when combined to MBCP biomaterial keep their hypoimmunogenicity. PBMC from healthy individual (\#713) were used as responder cells towards either BM-derived MSCs (MSC-BM) or AT-derived MSCs (MSC-AT) that were pretreated with IFN $\gamma$ and TNF $\alpha$ (IFN $\gamma / T N F \alpha)$ or not and used as stimulating cells at the responder: stimulator ratio of 1:0.2. MSCs were combined to MBCP biomaterial (MBCP) or not (No MBCP). Irradiated LCL* were used as positive control of T cell alloproliferation. Results are given as mean cpm \pm s.e.m.; one representative experiment is shown.

MSCs as stimulating cells. The osteodifferentiation process was validated through the upregulation of ALP expression in osteodifferentiated MSCs (Figures 3(a), 3(b), 4(a), and 4(b)). Results show that both BM-derived and AT-derived MSCs committed to osteodifferentiation are still hypoimmunogenic whether they are pretreated or not with IFN- $\gamma$ and TNF- $\alpha$ (Figures 3(c) and 4(c) and Tables 1 and 2). Then, we looked at whether combination of biomaterial (i.e., MBCP) with MSCs alters their immunogenicity. No differences were found between standard 2D-coculture conditions (MSC + PBMC) and 3D-coculture conditions (MSC + MBCP + PBMC). One representative allogenic combination is shown (Figure 5) for which the mean percentage of $\mathrm{T}$ cell alloproliferation is presented in Table 3 ( $n=3$ healthy donors). It is of note that the addition of MBCP to BM-derived MSCs treated or not with cytokines modifies slightly their immunogenicity, although no statistical difference was observed between both conditions $(P>0.1)$ (Table 3). Consequently, we can conclude that both the osteodifferentiation process and the presence of biomaterial (MBCP) did not abrogate the hypoimmunogenicity of MSCs.

Notably, expression of the tolerogenic soluble HLA-G5 protein was observed in MSCs derived from BM and AT and could be enhanced following treatment with IFN- $\gamma$ and TNF- $\alpha$ (Figures 1(b) and 2(b)). Such enhanced expression of HLA-G by IFN- $\gamma$ treatment was previously reported for other cell types such as monocytes [15], bronchial epithelial cells [16], thymic epithelial cells [17], and various tumor cells [18]. Nevertheless, HLA-G expression levels varied among the various batches of MSCs tested (data not shown). In 


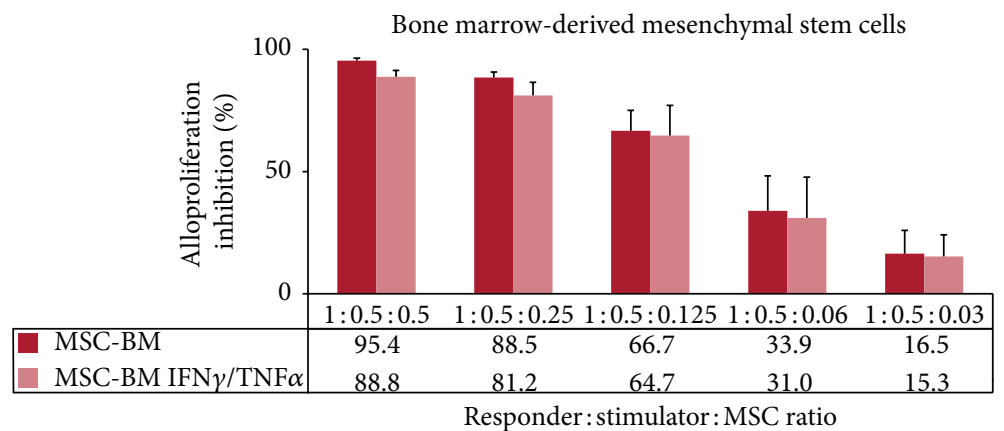

(a)

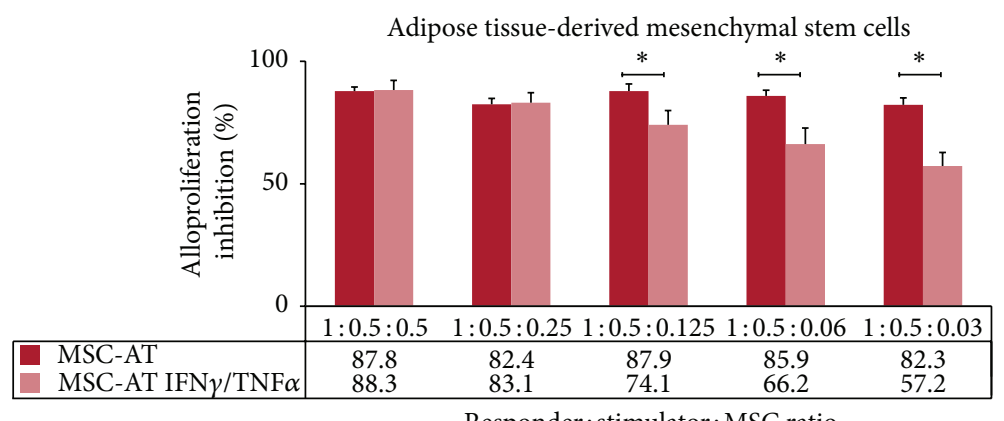

(b)

Figure 6: Both BM- andAT-derived MSCs display immunosuppressive properties in a dose-dependent manner. PBMC from healthy individuals were used as responder cells towards irradiated LCL* ${ }^{*}$ used as stimulating cells in presence of either (a) BM-derived MSCs (MSC$\mathrm{BM}$ ) or (b) AT-derived MSCs (MSC-AT) that were pretreated with IFN $\gamma$ and TNF $\alpha$ (IFN $\gamma / \mathrm{TNF} \alpha$ ) or not and used as third-party cells at various responder: stimulator: MSC ratios. Results are given as mean percentage of alloproliferation inhibition \pm s.e.m. according to the maximal alloproliferation observed with $\mathrm{PBMC}+\mathrm{LCL}^{*}$ using PBMC from 4 and 6 distinct healthy donors in BM- and AT-derived MSCs experiments, respectively.

TABLE 1: MSC from BM after osteodifferentiation and licensing by IFN $-\gamma$ and TNF- $\alpha$ do not induce PBMC proliferation in allogenic conditions.

\begin{tabular}{lcccc}
\hline $\begin{array}{l}\mathrm{R}: \mathrm{S}^{\mathrm{a}} \\
\text { ratio }\end{array}$ & MSC-BM & $\begin{array}{c}\text { MSC-BM } \\
\text { IFN- } \gamma / \text { TNF- } \alpha\end{array}$ & Os-MSC-BM & $\begin{array}{c}\text { Os-MSC-BM } \\
\text { IFN- } \gamma / \text { TNF- } \alpha\end{array}$ \\
\hline $1: 0.5$ & $4.3 \pm 2.1^{\mathrm{b}}$ & $1.1 \pm 0.3$ & $3.3 \pm 0.3$ & $1.7 \pm 0.2$ \\
$1: 0.25$ & $4.5 \pm 1.4$ & $1.0 \pm 0.3$ & $3.1 \pm 0.1$ & $1.8 \pm 0.5$ \\
$1: 0.12$ & $5.3 \pm 1.9$ & $1.1 \pm 0.4$ & $3.2 \pm 1.0$ & $2.3 \pm 1.2$ \\
$1: 0.06$ & $5.4 \pm 2.4$ & $1.4 \pm 0.5$ & $2.6 \pm 1.1$ & $3.9 \pm 3.1$ \\
$1: 0.03$ & $4.3 \pm 1.7$ & $1.4 \pm 0.5$ & $2.2 \pm 0.2$ & $2.1 \pm 1.2$ \\
\hline
\end{tabular}

${ }^{\mathrm{a}} \mathrm{PBMC}$ from healthy individuals were used as responder cells towards BMderived MSCs pretreated with IFN- $\gamma$ and TNF- $\alpha$ (MSC-BM IFN- $\gamma /$ TNF$\alpha$ ) or not (MSC-BM) as stimulating cells at various responder: stimulator $(\mathrm{R}: \mathrm{S})$ ratios. Similar experiments were performed with BM-derived MSCs committed to osteodifferentiation process and pretreated with IFN $-\gamma$ and TNF- $\alpha$ (Os-MSC-BM IFN- $\gamma /$ TNF- $\alpha$ ) or not (Os-MSC-BM) as stimulating cells.

${ }^{b}$ Data are mean \pm s.e.m. of alloproliferation percentage obtained with 5 and 3 distinct healthy donors for MSC-BM and Os-MSC-BM experiments, respectively. This percentage is calculated considering PBMC stimulated with $\mathrm{LCL}^{*}$ as $100 \%$ alloproliferation.

agreement with recent findings, we observed enhanced HLAG expression by MSCs following osteodifferentiation (Figures 3(a), 3(b), and 4(b)) [13].
TABLE 2: MSC from AT after osteodifferentiation and licensing by IFN $-\gamma$ and TNF- $\alpha$ do not induce PBMC proliferation in allogenic conditions.

\begin{tabular}{lcccc}
\hline $\begin{array}{l}\mathrm{R}: \mathrm{S}^{\mathrm{a}} \\
\text { ratio }\end{array}$ & MSC-AT & $\begin{array}{c}\text { MSC-AT } \\
\text { IFN- } \gamma / \text { TNF- } \alpha\end{array}$ & Os-MSC-AT & $\begin{array}{c}\text { Os-MSC-AT } \\
\text { IFN- } \gamma / \text { TNF- } \alpha\end{array}$ \\
\hline $1: 0.5$ & $5.6 \pm 1.0^{\mathrm{b}}$ & $7.2 \pm 3.1$ & $1.1 \pm 0.2$ & $0.9 \pm 0.6$ \\
$1: 0.25$ & $5.5 \pm 1.5$ & $5.2 \pm 2.6$ & $1.3 \pm 0.3$ & $0.8 \pm 0.5$ \\
$1: 0.12$ & $5.3 \pm 1.5$ & $4.7 \pm 2.9$ & $1.0 \pm 0.3$ & $1.1 \pm 0.8$ \\
$1: 0.06$ & $5.3 \pm 2.0$ & $5.5 \pm 3.0$ & $1.0 \pm 0.2$ & $1.3 \pm 1.0$ \\
$1: 0.03$ & $4.6 \pm 1.1$ & $3.9 \pm 2.0$ & $1.4 \pm 0.3$ & $1.8 \pm 1.3$ \\
\hline
\end{tabular}

${ }^{a} \mathrm{PBMC}$ from healthy individuals were used as responder cells towards ATderived MSCs pretreated with IFN- $\gamma$ and TNF- $\alpha$ (MSC-AT IFN- $\gamma /$ TNF$\alpha$ ) or not (MSC-AT) as stimulating cells at various responder: stimulator $(\mathrm{R}: \mathrm{S})$ ratios. Similar experiments were performed with AT-derived MSCs committed to osteodifferentiation process and pretreated with IFN- $\gamma$ and TNF- $\alpha$ (Os-MSC-AT IFN- $\gamma /$ TNF- $\alpha$ ) or not (Os-MSC-AT) as stimulating cells.

${ }^{\mathrm{b}}$ Data are mean \pm s.e.m. of alloproliferation percentage obtained with 4 and 3 distinct healthy donors for MSC-AT and Os-MSC-AT experiments, respectively. This percentage is calculated considering PBMC stimulated with LCL $^{*}$ as $100 \%$ alloproliferation.

Then, we investigated the immunomodulatory properties of MSCs as third-party cells in MLR. Results showed that both BM-derived and AT-derived MSCs display immunosuppressive properties in a dose-response manner (Figure 6). It is 


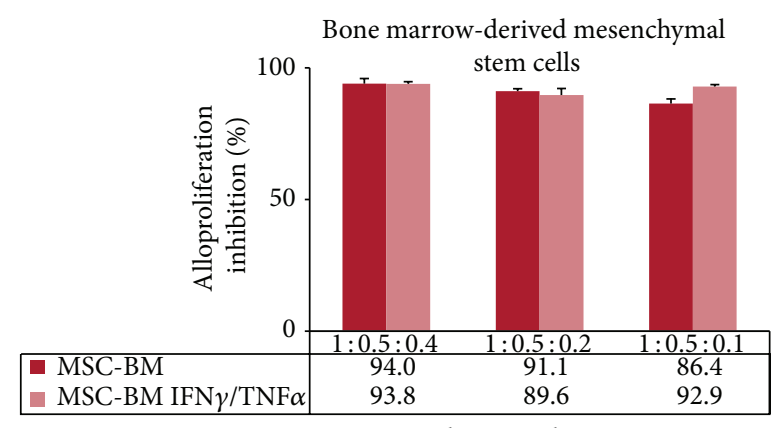

Responder: stimulator: MSC ratio
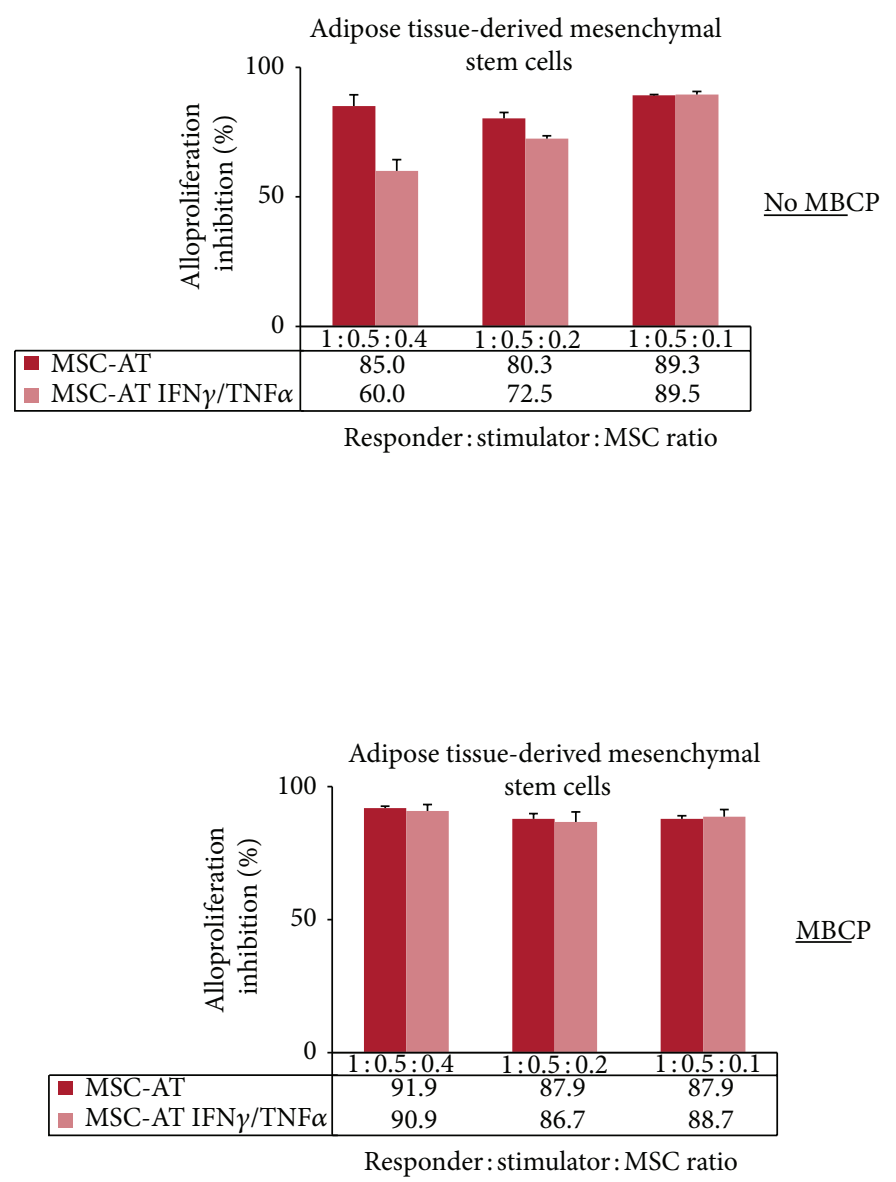

FIGURE 7: Both BM- andAT-derived MSCs when combined to MBCP biomaterial keep their immunosuppressive properties. PBMC from healthy individuals were used as responder cells towards irradiated $\mathrm{LCL}^{*}$ used as stimulating cells in presence of either BM-derived MSCs (MSC-BM) or AT-derived MSCs (MSC-AT) that were pretreated with IFN $\gamma$ and TNF $\alpha$ (IFN $\gamma / \mathrm{TNF} \alpha$ ) or not and used as third-party cells at various responder: stimulator : MSC ratios. MSCs were combined to MBCP biomaterial (MBCP) or not (No MBCP). Results are given as mean percentage of alloproliferation inhibition \pm s.e.m. when compared to PBMC+LCL* using PBMC from 3 distinct healthy donors.

TABLE 3: Immunogenicity of MSC from bone marrow and adipose tissue combined with MBCP biomaterial and licensing by IFN- $\gamma$ and TNF- $\alpha$.

\begin{tabular}{lcccc}
\hline & MSC-BM & $\begin{array}{c}\text { MSC-BM } \\
\text { IFN- } \gamma / \text { TNF- } \alpha\end{array}$ & MSC-AT & $\begin{array}{c}\text { MSC-AT } \\
\text { IFN- } \gamma / \text { TNF- } \alpha\end{array}$ \\
\hline No MBCP & $7.5 \pm 2.2^{\mathrm{b}}$ & $9.8 \pm 4.0$ & $13.9 \pm 3.9$ & $23.0 \pm 5.2$ \\
MBCP & $51.9 \pm 14.1$ & $41.1 \pm 11.6$ & $8.1 \pm 0.8$ & $11.0 \pm 0.8$ \\
\hline
\end{tabular}

${ }^{\mathrm{a}} \mathrm{PBMC}$ from healthy individuals were used as responder cells towards BMor AT-derived MSCs pretreated with IFN- $\gamma$ and TNF- $\alpha$ or not as stimulating cells at $1: 0.2$ responder : stimulator ratio.

${ }^{\mathrm{b}}$ Data are mean \pm s.e.m. of alloproliferation percentage obtained with 3 distinct healthy donors. This percentage is calculated considering PBMC stimulated with $\mathrm{LCL}^{*}$ as $100 \%$ alloproliferation.

of note that (i) AT-derived MSCs are more potent at low doses compared to BM-derived MSCs $(P<0.05)$ (Figures 6(a) and $6(\mathrm{~b}))$ and (ii) licensing with cytokines reduces significantly the immunosuppressive properties of AT-derived MSCs $(P<0.05)$ (Figure 6(b)). When combined to biomaterial (MBCP), both BM-derived and AT-derived MSCs still exert immunosuppressive properties as they greatly inhibit $\mathrm{T}$ cell alloproliferation with or without being seeded with $\mathrm{MBCP}$ (Figure 7). Although not statistically significant, addition of $\mathrm{MBCP}$ reduces the immunomodulatory properties of BMderived MSCs at high responder: stimulator: MSC ratios. This could be due to steric hindrance when high numbers of cells are used. Indeed, such MBCP effect is no longer observed at low ratios (Figure 7). Also, both BM-derived and AT-derived MSCs when committed to preosteoblastic MSCs inhibit T cell alloproliferation and remain thus able to induce a tolerogenic microenvironment (Figure 8). Licensing with IFN- $\gamma$ and TNF- $\alpha$ did not modify such MSC-derived immunosuppression (Figures 7 and 8). It is of note that once osteodifferentiated AT-derived MSCs are more potent at low doses compared to BM-derived MSCs as they display higher immunosuppressive effects $(P<0.05)$ (Figure 8). Such higher immunomodulatory capacity of adipose tissuederived multipotent stromal cells compared to their bone marrow-derived counterparts has been previously reported [19].

Our present results are in agreement with previous reports showing that differentiation of stem cells does not 




(a)

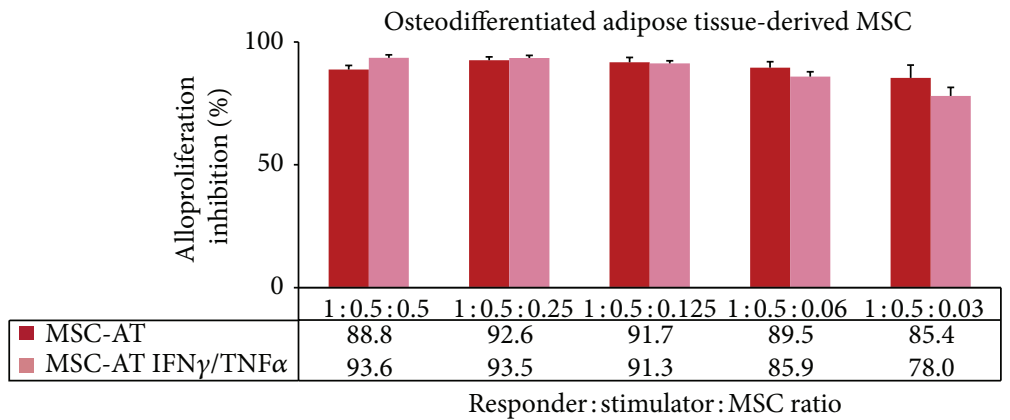

(b)

FIGURE 8: Both BM- andAT-derived MSCs when committed to osteodifferentiation process keep their immunosuppressive properties. PBMC from healthy individuals were used as responder cells towards irradiated LCL* used as stimulating cells in presence of either osteodifferentiated BM-derived MSCs (Os-MSC-BM) or AT-derived MSCs (Os-MSC-AT) that were pretreated with IFN $\gamma$ and TNF $\alpha$ $(\mathrm{IFN} \gamma / \mathrm{TNF} \alpha)$ or not and used as third-party cells at various responder : stimulator: MSC ratios. Results are given as mean percentage of alloproliferation inhibition \pm s.e.m. when compared to PBMC+LCL* using PBMC from 3 and 5 distinct healthy donors in BM- and ATderived MSCs experiments, respectively.

alter their low immunogenicity and immunomodulatory properties. For instance, human amniotic epithelial cells, which have stem cell-like properties, retain their immunosuppressive functions after differentiation into hepatocytelike cells [20]. Also, human Wharton's jelly-derived MSCs maintain the expression of immunomodulatory molecules, such as HLA-G, when subjected to osteogenic differentiation in vitro [21].

In conclusion, MSCs from BM or AT display tolerogenic properties which are maintained following osteodifferentiation process or addition of biomaterial and may thus be considered as allogenic sources for regenerating bone defects in orthopaedic and maxillofacial surgery [22].

\section{Conflict of Interests}

The authors declare that there is no conflict of interests regarding the publication of this paper.

\section{Acknowledgments}

This project has received funding from the European Union's Seventh Programme for research, technological development, and demonstration under Grant agreement no. 241879.

\section{References}

[1] R. Langer and J. P. Vacanti, “Tissue engineering," Science, vol. 260, no. 5110, pp. 920-926, 1993.

[2] C. Laurencin, Y. Khan, and S. F. El-Amin, "Bone graft substitutes," Expert Review of Medical Devices, vol. 3, no. 1, pp. 49-57, 2006.

[3] H. Petite, V. Viateau, W. Bensaïd et al., "Tissue-engineered bone regeneration," Nature Biotechnology, vol. 18, no. 9, pp. 959-963, 2000.

[4] M. Di Trapani, G. Bassi, M. Ricciardi et al., "Comparative study of immune regulatory properties of stem cells derived from different tissues," Stem Cells and Development, vol. 22, pp. 29903002, 2013.

[5] A. Uccelli, V. Pistoia, and L. Moretta, "Mesenchymal stem cells: a new strategy for immunosuppression?" Trends in Immunology, vol. 28, no. 5, pp. 219-226, 2007.

[6] M. Najar, G. Raicevic, H. Fayyad-Kazan et al., "Immune-related antigens, surface molecules and regulatory factors in humanderived mesenchymal stromal cells: the expression and impact of inflammatory priming," Stem Cell Reviews, vol. 8, pp. 11881198, 2012.

[7] R. Rizzo, D. Campioni, M. Stignani et al., "A functional role for soluble HLA-G antigens in immune modulation mediated by mesenchymal stromal cells," Cytotherapy, vol. 10, no. 4, pp. 364375, 2008. 
[8] F. Morandi, L. Raffaghello, G. Bianchi et al., "Immunogenicity of human mesenchymal stem cells in HLA-class I-restricted Tcell responses against viral or tumor-associated antigens," Stem Cells, vol. 26, no. 5, pp. 1275-1287, 2008.

[9] A. Naji, N. Rouas-Freiss, A. Durrbach, E. D. Carosella, L. Sensebe, and F. Deschaseaux, "Concise review: combining human leukocyte antigen $G$ and mesenchymal stem cells for immunosuppressant biotherapy," Stem Cells, vol. 31, pp. 22962303, 2013.

[10] G. La Rocca and R. Anzalone, "Perinatal stem cells revisited: directions and indications at the crossroads between tissue regeneration and repair," Current Stem Cell Research \& Therapy, vol. 8, pp. 2-5, 2013.

[11] Z. Selmani, A. Naji, E. Gaiffe et al., "HLA-G is a crucial immunosuppressive molecule secreted by adult human mesenchymal stem cells," Transplantation, vol. 87, no. 9, supplement, pp. S62S66, 2009.

[12] Z. Selmani, A. Naji, I. Zidi et al., "Human leukocyte antigenG5 secretion by human mesenchymal stem cells is required to suppress T lymphocyte and natural killer function and to induce $\mathrm{CD} 4^{+} \mathrm{CD} 25^{\text {high }} \mathrm{FOXP3}^{+}$regulatory T cells," Stem Cells, vol. 26, no. 1, pp. 212-222, 2008.

[13] F. Deschaseaux, J. Gaillard, A. Langonne et al., "Regulation and function of immunosuppressive molecule human leukocyte antigen G5 in human bone tissue," The FASEB Journal, vol. 27, pp. 2977-2987, 2013.

[14] M. Krampera, "Mesenchymal stromal cell licensing: a multistep process," Leukemia, vol. 25, no. 9, pp. 1408-1414, 2011.

[15] P. Moreau, F. Adrian-Cabestre, C. Menier et al., "IL-10 selectively induces HLA-G expression in human trophoblasts and monocytes," International Immunology, vol. 11, no. 5, pp. 803811, 1999.

[16] O. Brugière, G. Thabut, M. Pretolani et al., "Immunohistochemical study of HLA-G expression in lung transplant recipients," American Journal of Transplantation, vol. 9, no. 6, pp. 1427-1438, 2009.

[17] S. Lefebvre, S. Berrih-Aknin, F. Adrian et al., "A specific interferon (IFN)-stimulated response element of the distal HLA-G promoter binds IFN-regulatory factor 1 and mediates enhancement of this nonclassical class I gene by IFN-beta," The Journal of Biological Chemistry, vol. 276, no. 9, pp. 6133-6139, 2001.

[18] N. Rouas-Freiss, S. Bruel, C. Menier, C. Marcou, P. Moreau, and E. D. Carosella, "Switch of HLA-G alternative splicing in a melanoma cell line causes loss of HLA-G1 expression and sensitivity to NK lysis," International Journal of Cancer, vol. 117, no. 1, pp. 114-122, 2005.

[19] S. M. Melief, J. J. Zwaginga, W. E. Fibbe, and H. Roelofs, "Adipose tissue-derived multipotent stromal cells have a higher immunomodulatory capacity than their bone marrow-derived counterparts," Stem Cells Translational Medicine, vol. 2, pp. 455463, 2013.

[20] J. Y. Tee, V. Vaghjiani, Y. H. Liu, P. Murthi, J. Chan, and U. Manuelpillai, "Immunogenicity and immunomodulatory properties of hepatocyte-like cells derived from human amniotic epithelial cells," Current Stem Cell Research \& Therapy, vol. 8, pp. 91-99, 2013.

[21] G. La Rocca, M. Lo Iacono, T. Corsello, S. Corrao, F. Farina, and R. Anzalone, "Human Wharton's jelly mesenchymal stem cells maintain the expression of key immunomodulatory molecules when subjected to osteogenic, adipogenic and chondrogenic differentiation in vitro: new perspectives for cellular therapy," Current Stem Cell Research \& Therapy, vol. 8, pp. 100-113, 2013.

[22] C. Menard and K. Tarte, "Immunoregulatory properties of clinical grade mesenchymal stromal cells: evidence, uncertainties, and clinical application," Stem Cell Research \& Therapy, vol. 4, article 64, 2013. 


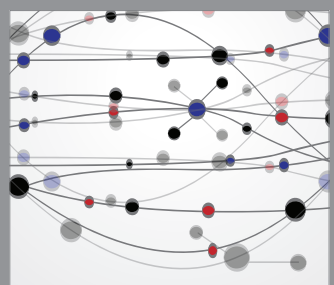

The Scientific World Journal
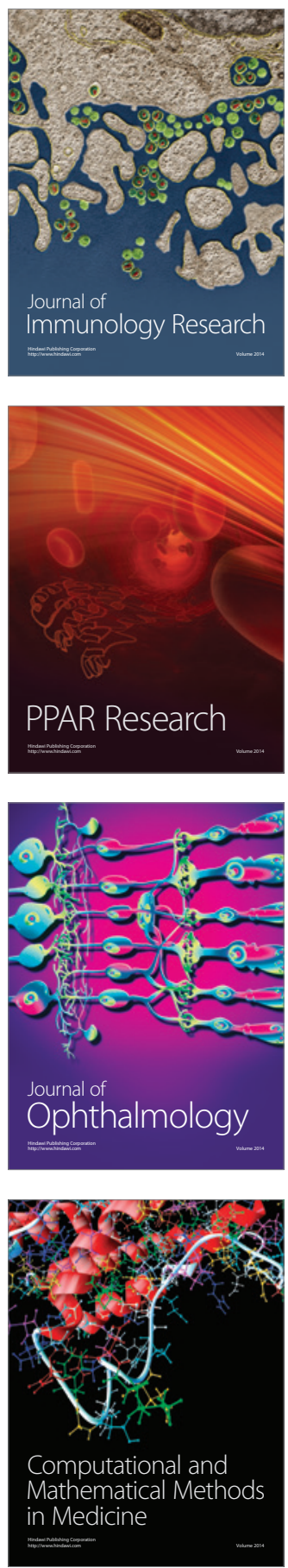



Gastroenterology

Research and Practice
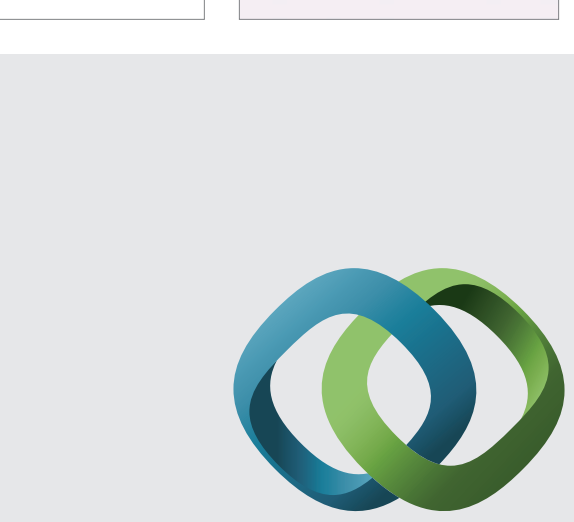

\section{Hindawi}

Submit your manuscripts at

http://www.hindawi.com
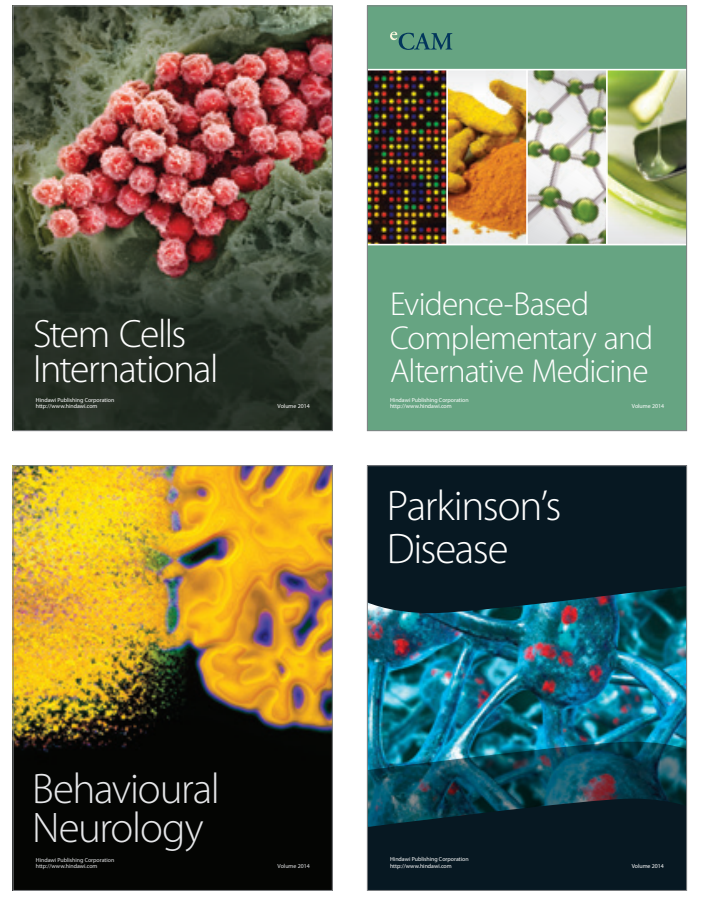
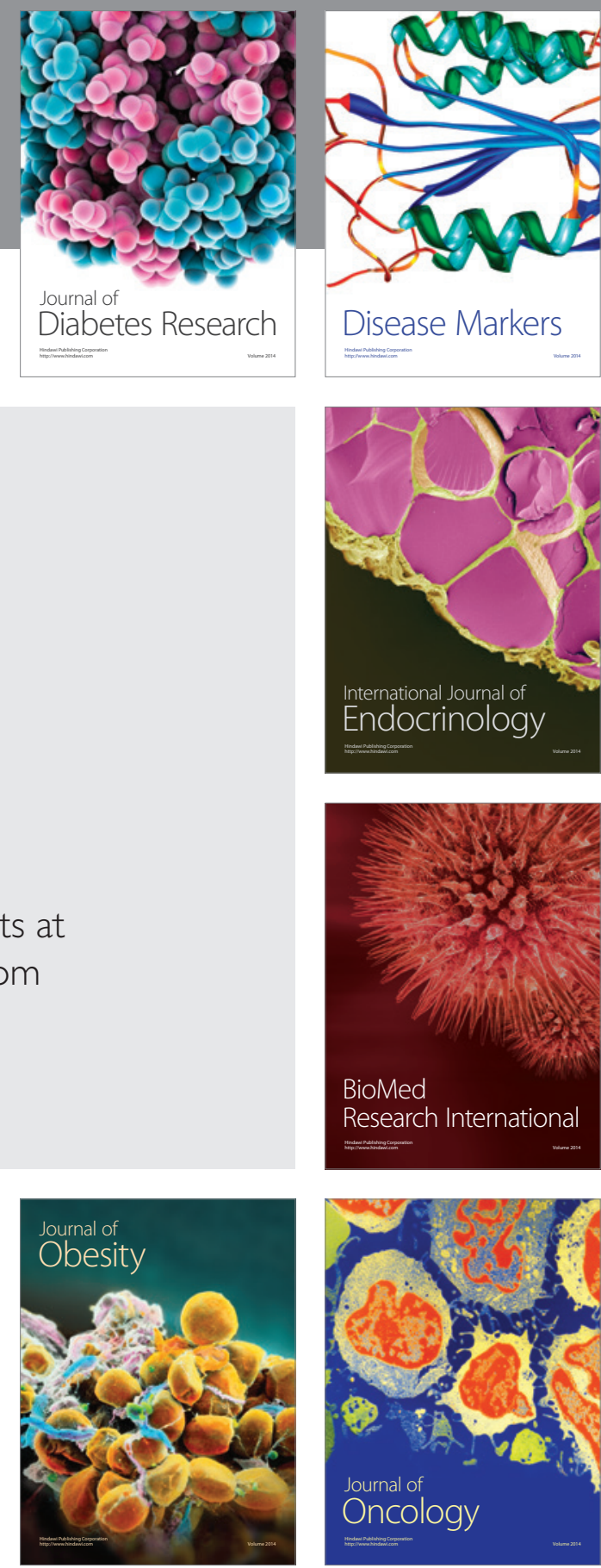

Disease Markers
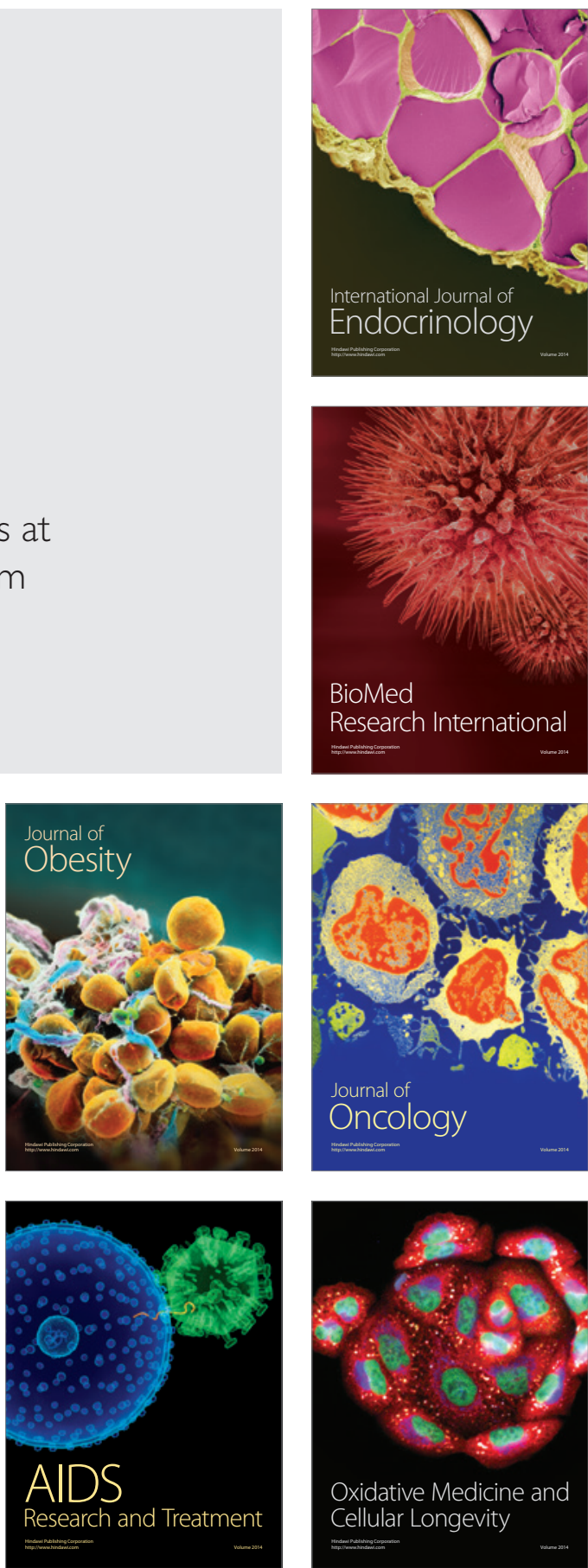